\title{
Calculation of the Thermal Properties (and Their Uncertainties) of Strawberry During Its Cooling Under Natural Convection
}

\author{
W. P. Silva ${ }^{1}$, C. M. D. P. S. e Silva ${ }^{1}$, J. P. Gomes ${ }^{2}$, N. C. Santos $^{2}$, A. J. M. Queiroz ${ }^{2} \&$ R. M. F. de Figuiredo ${ }^{2}$ \\ ${ }^{1}$ Department of Physics, Federal University of Campina Grande, Campina Grande, PB, Brazil \\ ${ }^{2}$ Department of Agricultural Engineering, Federal University of Campina Grande, Campina Grande, PB, Brazil \\ Correspondence: Wilton Pereira da Silva, Department of Physics, Federal University of Campina Grande, R. \\ Aprígio Veloso, 882, Campina Grande, PB, Brazil. Tel: 55-839-692-7630. E-mail: wiltonps@uol.com.br
}

\author{
Received: January 20, $2019 \quad$ Accepted: February 26, $2019 \quad$ Online Published: April 15, 2019 \\ doi:10.5539/jas.v11n5p114 URL: https://doi.org/10.5539/jas.v11n5p114
}

The research is financed by Conselho Nacional de Desenvolvimento Cientifico e Tecnológico-CNPq.

\begin{abstract}
Many times, the thermal properties of a product are determined but their uncertainties (and, mainly, the covariance matrix) are not provided. Thus, in the simulations, it is not possible to establish a confidence band for a transient state described through the values obtained for these properties. In this article, a model was proposed to determine thermal diffusivity and convective heat transfer coefficient, providing the above-mentioned lack of information, for a product with spherical geometry during its cooling. The proposed model involved: 1) an experimental data set of the cooling kinetics in a point within the product; 2) a one-dimensional numerical solution of the heat conduction equation; 3) an optimizer based on the Levenberg-Marquardt algorithm to determine the thermal properties, their uncertainties, and the covariance between the parameters. Model was applied for determining thermal properties of strawberries, using an equivalent sphere to represent the geometry of the product, and the obtained results were compatible with the literature results.
\end{abstract}

Keywords: direct problem, inverse problem, finite volume, thermal diffusivity, convective heat transfer coefficient

\section{Introduction}

In the literature, several methods are found to determine the thermal properties of agricultural products during their cooling. One of these methods is based on the cooling kinetics of a point within the product, usually the central point (Dincer, 1995; Silva et al., 2011, 2012; Erdogdu et al., 2014; Da Silva et al., 2018). In this method, a solution of the heat conduction equation is used to describe the experimental data set of the cooling kinetics, and the thermal properties are determined using some type optimization. As example, Dincer (1995) has used an analytical solution of the heat conduction equation, in which the series that represents the solution was truncated and only its first term was used in the calculation of the thermal parameters, using non-linear regression. In another example, Erdogdu et al. (2014) have also used with success a single term of the series for representing the analytical solution of one-dimensional heat conduction equation. The authors disregarded, by graphical observation of the logarithm applied on the dimensionless temperature versus time, the first experimental points through the identification of the linear portion of the graph. In this case in which logarithm is applied on the dimensionless temperatures, a simple linear regression enables to determine the thermal properties. Silva et al. (2012) have also used only the first term of the series for the non-linear regression, but these authors have repeated the procedure several times, eliminating the first experimental point; after that the authors eliminate two experimental points, etc. and, in each case, they determined the thermal properties by curve fitting. After that, the parameters determined in each case were used to calculate the corresponding chi-square, involving all the experimental points, in which more than 100 terms of the series were used. Finally, the authors compared all of the chi-squares, considering as optimal values for the parameters those corresponding to the lowest chi-square. To this algorithm the authors gave the name OREP (Optimal Removal of Experimental Points).

Recently, the LS Optimizer program, based on the Levenberg-Marquardt algorithm (Levenberg, 1944; Marquardt, 1963), was made available free on the Internet by the first two authors of the present article. The 
purpose of this program was to determine parameters of differential equations and complex functions, including average values, their uncertainties and the covariance matrix (http://zeus.df.ufcg.edu.br/labfit/LS.htm). Da Silva et al. (2018) used this optimizer and an analytical solution of the diffusion equation (first 200 terms) for determining the thermal properties of banana with peel, their uncertainties and the covariance matrix. The authors have reported good results for the calculated parameters using this model involving LS Optimizer.

An observation in the literature (Sweat, 1986; ASHRAE, 1993; Fricke \& Becker, 2001; Da Silva et al., 2010, 2011; Silva et al., 2011, 2012) makes it possible to realize that it is not so common to determine thermal properties of agricultural products, including their uncertainties and the covariance matrix, when only one experimental data set is available. However, LS Optimizer enables these calculations and, in this sense, the objectives of this article are defined in the following.

The main objective of this article was to propose a model to determine thermal properties of agricultural products, which may be considered as a sphere, during their cooling. The model have involved the following characteristics: 1) a solver to describe the direct problem, in order to simulate the kinetics cooling of a point within the sphere equivalent to the real geometry of the product; 2) an experimental data set of the cooling kinetics of a point within the equivalent sphere; 3) an optimization program for the inverse problem, which consists in determining of the thermal properties (their uncertainties and the covariance matrix) for the product. The model was used to study strawberry cooling, determining not only its thermal properties, but also simulating the process.

\section{Material and Methods}

\subsection{Diffusion Equation}

In spherical coordinates, the one-dimensional heat conduction equation is written in the following way:

$$
\frac{\partial\left(\rho c_{p} T\right)}{\partial t}=\frac{1}{r^{2}} \frac{\partial}{\partial r}\left(r^{2} k \frac{\partial T}{\partial r}\right)
$$

where, $T$ is the temperature, $t$ is the cooling time, $\rho$ is the density of the product, $c_{p}$ is the specific heat, $r$ defines a position within the equivalent sphere (with respect to the center) and $k$ is the thermal conductivity. On the other hand, in a cooling process, the temperature variation is usually equal or less than $25^{\circ} \mathrm{C}$ and, in this case, $\rho$ and $c_{p}$ can be assumed as constant properties. Thus, dividing Equation (1a) by the product $\rho c_{p}$, it is obtained:

$$
\frac{\partial T}{\partial t}=\frac{1}{r^{2}} \frac{\partial}{\partial r}\left(r^{2} \alpha \frac{\partial T}{\partial r}\right)
$$

where, $\alpha=k /\left(\rho c_{p}\right)$ is the thermal diffusivity. For the cooling process, the boundary condition is, usually, of the third kind, and it can be expressed by the following equations:

$$
-k \frac{\partial T}{\partial r}=h_{H}\left(T_{b}-T_{\infty}\right)
$$

or, dividing by $\rho c_{p}$,

$$
-\alpha \frac{\partial T}{\partial r}=h\left(T_{b}-T_{\infty}\right)
$$

where, $h=h_{H} /\left(\rho c_{p}\right)$ is the convective heat transfer coefficient, $T_{\mathrm{b}}$ is the temperature at the boundary, $T_{\infty}$ is the value of temperature of the air in which the strawberry fruit is cooled, and $h_{H}$ is the heat transfer coefficient.

\subsection{Direct Problem: Numerical Solution to Create a Solver}

Equation (1b), subjected to the boundary condition defined by Equation (2b), was solved by using the finite volume method, with a fully implicit formulation (Patankar, 1980). Figure 1 presents a sphere and its uniform one-dimensional mesh, in which the control volumes have a common thickness given by $\Delta r$; and the control volume number " $i$ " has a nodal point " $P$ ". 

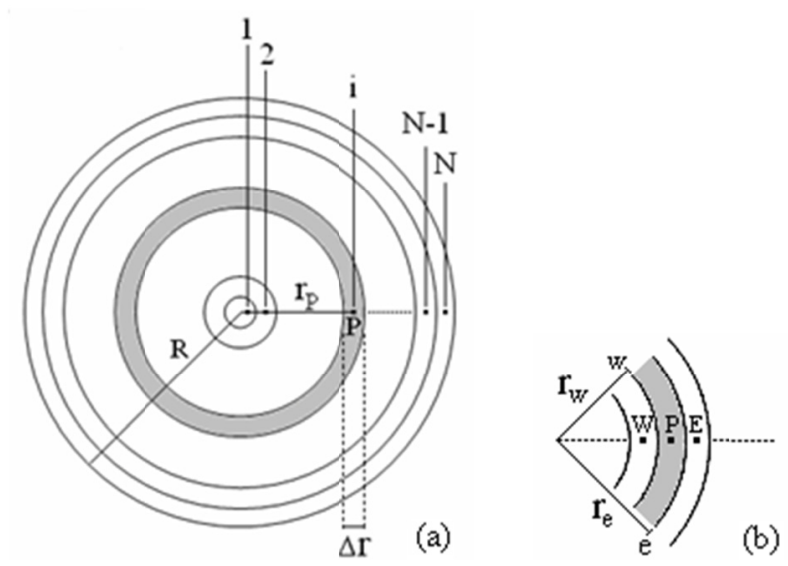

Figure 1. (a) Uniform mesh: $\mathrm{N}$ control volumes with thickness $\Delta r$; (b) Control volume $\mathrm{P}$ and its neighbors to west (W) and to east (E)

Integrating Equation (1b) in the space $\left(4 \pi r_{P}^{2} \Delta r\right)$ and time $(\Delta t)$, Equation (3) is obtained:

$$
\frac{T_{P}-T_{P}^{0}}{\Delta t} r_{P}^{2} \Delta r=\left.r_{e}^{2} \alpha \frac{\partial T}{\partial r}\right|_{e}-\left.r_{w}^{2} \alpha \frac{\partial T}{\partial r}\right|_{w}
$$

The superscript 0 means initial time $(t)$ and its absence means final time of the time step, $(\mathrm{t}+\Delta t)$. The indexes " $P$ ", " $e$ " and " $w$ " refer, respectively, to the nodal point, east interface and west interface of the control volume. On the other hand, discretizing Equation (2b), Equation (4) is obtained:

$$
-\alpha \frac{T_{b}-T_{P}}{\Delta r / 2}=h\left(T_{b}-T_{\infty}\right)
$$

and, therefore,

$$
T_{b}=\frac{T_{\infty}+\frac{\alpha}{h \Delta r / 2} T_{P}}{1+\frac{\alpha}{h \Delta r / 2}}
$$

An internal control volume has neighbors to west and east as it is shown in Figure 1, from the control volume 2 to N-1. For this type of control volume, Equation (3) results in:

$$
A_{p} T_{P}=A_{w} T_{W}+A_{e} T_{E}+B
$$

with

$$
A_{p}=\frac{r_{P}^{2} \Delta r}{\Delta t}+\frac{r_{e}^{2}}{\Delta r} \alpha+\frac{r_{w}^{2}}{\Delta r} \alpha ; A_{w}=\frac{r_{w}^{2}}{\Delta r} \alpha ; A_{e}=\frac{r_{e}^{2}}{\Delta r} \alpha ; B=\frac{r_{P}^{2} \Delta r}{\Delta t} T_{P}^{0}
$$

Due to the symmetry condition, Equation (3), for the control volume 1, becomes:

$$
A_{p} T_{P}=A_{e} T_{E}+B
$$

where,

$$
A_{p}=\frac{r_{P}^{2} \Delta r}{\Delta t}+\frac{r_{e}^{2}}{\Delta r} \alpha ; A_{e}=\frac{r_{e}^{2}}{\Delta r} \alpha ; B=\frac{r_{P}^{2} \Delta r}{\Delta t} T_{P}^{0}
$$

The combination of Equations (3) and (4) for the control volume $\mathrm{N}$ (boundary) gives the following result:

$$
A_{p} T_{P}=A_{w} T_{W}+B
$$

where,

$$
A_{p}=\frac{r_{P}^{2} \Delta r}{\Delta t}+\frac{r_{e}^{2} \alpha}{\Delta r / 2+\alpha / h}+\frac{r_{w}^{2} \alpha}{\Delta r} ; A_{w}=\frac{r_{w}^{2} \alpha}{\Delta r} ; \quad B=\frac{r_{P}^{2} \Delta r}{\Delta t} T_{P}^{0}+\frac{r_{e}^{2} \alpha}{\Delta r / 2+\alpha / h} T_{\infty}
$$

In this article, for each time step (from $t$ to $t+\Delta t$ ), the system of equations defined by Equations (6), (8) and (10) was solved by the TDMA (Three-Diagonal Matrix Algorithm) method, as presented, for instance, by Press et al. (1996). 


\subsection{Inverse Problem: Optimization}

In order to determine $\alpha$ and $h$, including their uncertainties, LS Optimizer Software was used. As above-mentioned, this software uses Levenberg-Marquardt algorithm (Levenberg, 1944; Marquardt, 1963) and executes the solver provided by the user for the direct problem, with the objective of determining the parameters $\alpha$ and $h$ that make it possible a simulation as close as possible to the experimental data set (minimum chi-square). According to the information available in the LS Optimizer manual, as long as the solver for the direct problem and the experimental data set are provided, this optimization software executes the solver in order to obtain the necessary information for the determination of parameters, using the statistical indicator chi-square as objective function: $\chi^{2}=\Sigma\left(T^{e x p}-T^{s i m}\right)^{2} / \sigma^{2}$. If the uncertainties of the experimental points $(\sigma)$ are not known at first, LS Optimizer determines the standard deviation associated with the optimization process using the expression $\sigma^{\prime}=$ $\left[\Sigma\left(T^{e x p}-T^{s i m}\right)^{2} /\left(N_{p}-n\right)\right]^{1 / 2}$ in which $\left(N_{p}-n\right)$ is the number of degrees of freedom for the optimization process. Hence, at the end of the iterative process, one can impose $\sigma=\sigma^{\prime}$ and recalculate the covariance matrix, which provides the uncertainties of the parameters and the correlation between them. Thus, starting from initial values $\alpha_{0}$ and $h_{0}$, the optimizer corrects these parameters iteratively up to the convergence and, in the end, it delivers the optimal values for $\alpha$ and $h$, their uncertainties, and the covariance matrix.

\subsection{Experimental Data}

Silva et al. (2011) performed cooling of strawberry ( $X=90 \%$, wet basis) under natural convection, initially at $24.8{ }^{\circ} \mathrm{C}$, using a home refrigerator Brastemp with internal temperature set about $0.6{ }^{\circ} \mathrm{C}$ and relative humidity between 87 and 92\%. A thermocouple was inserted within the strawberry, for measuring the temperature at regular time interval during $140 \mathrm{~min}$, but its exact position was not determined during the experiment; and the group was placed inside the refrigerator. In the present article, the experimental data set was obtained by digitization, using the program xyExtract (V 5.1, 2011, http://zeus.df.ufcg.edu.br/labfit/index_xyExtract.htm). The real geometry of the strawberry was considered as an equivalent sphere, with radius $R=0.0167 \mathrm{~m}$, and this value was obtained by imposing same volume for the two geometries (real and sphere). For the equivalent sphere, the thermocouple position was estimated using optimization technique: $r=0.0091 \mathrm{~m}$.

Due to the robust method of optimization used by Silva et al. (2011) to determine thermal properties, the uncertainties of the obtained values were not calculated. In cooling processes, the calculation of only the average values of the parameters is a common procedure found in the literature (Sweat, 1986; ASHRAE, 1993; Fricke \& Becker, 2001; Silva et al., 2010, 2011). In the present work, the data set obtained by Silva et al. (2011) in the cooling experiment was used to determine not only the thermal properties of strawberry, by optimization, but also the uncertainties and the covariance between these parameters. Although it can't be assumed a priori that the error distribution to be obtained is a Gaussian distribution, as the experimental data set contains 61 points, in practical terms, the uncertainties to be obtained will define intervals with confidence at least close to $68.3 \%$ (Bevington \& Robinson, 1992; Taylor, 1997).

\section{Results and Discussion}

In this study, a fully implicit formulation was used for the numerical solution, due to the fact that this formulation ensures that such solution is unconditionally stable (Patankar, 1980). A preliminary study has shown that, in the simulations of a diffusion process in a small product with spherical geometry, 200 control volumes and 2000 steps of time are enough in order to disregard possible convergence errors for the iterative process (Da Silva et al., 2012). Thus, these refinements for the grid and time were used in all the calculations of this article. In the optimization process, the initial values were: $\alpha_{0}=1.5 \times 10^{-7}$ and $h_{0}=1.5 \times 10^{-6}$. The results obtained, as well as those obtained by Silva et al. (2011) are presented in Table 1 .

Table 1 . Thermal diffusivity and convective heat transfer coefficient for strawberry. The intervals were obtained with a confidence of $68.3 \%$

\begin{tabular}{lll}
\hline Thermal property & Present study & Silva et al. (2011) \\
\hline$\alpha\left(\mathrm{m}^{2} \mathrm{~s}^{-1}\right)$ & $(1.42 \pm 0.11) \times 10^{-7}$ & $1.42 \times 10^{-7}$ \\
$h\left(\mathrm{~ms}^{-1}\right)$ & $(3.196 \pm 0.039) \times 10^{-6}$ & $3.256 \times 10^{-6}$ \\
\hline
\end{tabular}

It should be remembered that the LS program minimizes the chi-square obtained by the deviations calculated by the differences between the simulated values and the values of the corresponding experimental points, and the minimum chi-square value is used to determine the uncertainties of each calculated parameter. This procedure is 
similar to the determination of uncertainties of parameters in the fitting of a function to experimental data through nonlinear regression (Levenberg, 1944; Marquardt, 1963).

An interesting fact to be highlighted in the results is the discrepancy between the values obtained in this article and in Silva et al. (2011): according to Table 1, for thermal diffusivity the discrepancy is zero; and for the convective heat transfer coefficient is only $1.9 \%$. In this sense, a comparison of the results indicates that the two models can be considered equivalent. Using the values obtained in the present article for thermal diffusivity and convective heat transfer coefficient, Figure 2 can be presented, showing the simulated curve and the experimental data set.

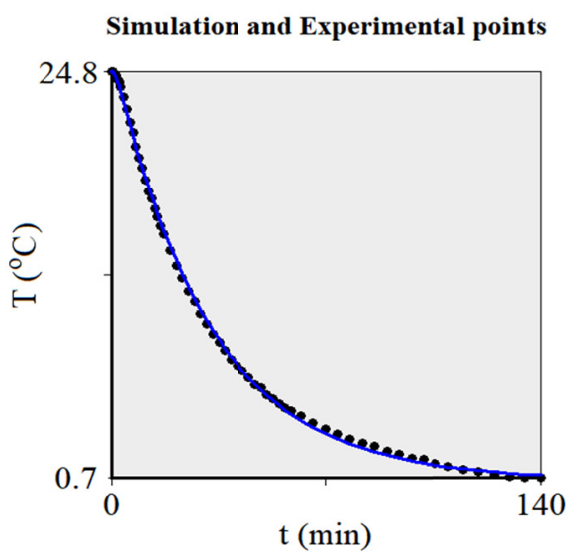

Figure 2. Cooling simulation using the values parameter provided in Table 1 and experimental data set

The statistical indicators for the optimization process, referring to Figure 2, were: chi-square equal to 2.4899 and determination coefficient equal to 0.9994 .

For the results obtained with the model proposed in this article, the percentage uncertainty in the calculation of the thermal diffusivity is $7.7 \%$, whereas in the convective heat transfer coefficient calculation it is only $1.2 \%$. Obviously, using just one experimental data set, this information only can be obtained through the proposed model, since Silva et al. (2011) model uses a robust method for the optimization process. Another important information is that referring to the correlation coefficient between the two properties. The proposed model enables to express this statistical parameter, equal to -0.9206 , which is a similar result that obtained by Da Silva et al. (2018) studying cooling of banana with peel, using LS Optimizer program. As the domain of this indicator is between -1 and 1 , it is noted that $\alpha$ and $h$ are highly correlated. In this case, as the correlation coefficient is negative, if one of them increases, other diminishes. This information is important in order to select adequate pairs $\alpha$ and $h$ in order to determine a confidence band that contain all possible results of the cooling kinetics. In this study, based on Table 1 and also on this discussion, were stipulated the following values: $\alpha=1.31 \times 10^{-7} \mathrm{~m}^{2}$ $\mathrm{s}^{-1} ; h=3.235 \times 10^{-6} \mathrm{~m} \mathrm{~s}^{-1}$ and $\alpha=1.53 \times 10^{-7} \mathrm{~m}^{2} \mathrm{~s}^{-1} ; h=3.175 \times 10^{-6} \mathrm{~m} \mathrm{~s}^{-1}$. Thus, the confidence band may be shown through Figure 3. 


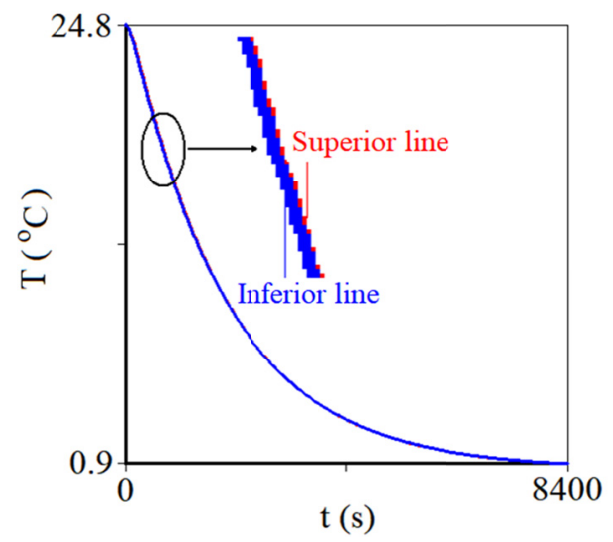

Figure 3. Confidence band for the cooling simulation using the confidence intervals of the parameters provided in Table 1

An observation of Figure 3 makes it possible to realize that the confidence band is very narrow, indicating that the experimental data set, as well as the results, can be considered as good. It is important to observe this result about the confidence band only it was possible due to the proposed model.

\section{Conclusion}

The proposed model presented results for the values of strawberry thermal properties compatible with the values obtained by other models available in the literature. In addition, the proposed model made it possible to determine the uncertainty of each studied thermal property, as well as the correlation coefficient between these properties. This made it possible to present a graph for the confidence band of the cooling kinetics of a point within the product. An observation of this graph allowed to conclude that the simulation of this cooling kinetics for strawberry fruit can be considered precise.

\section{Acknowledgements}

The first author would like to thank CNPq (Conselho Nacional de Desenvolvimento Científico e Tecnológico) for supporting this study and for his research grant (Processes Number 302480/2015-3).

\section{References}

ASHRAE. (1993). Handbook of Fundamentals. American Society of Heating, Refrigerating and Air Conditioning Engineers, Atlanta.

Bevington, P. R., \& Robinson, D. K. (1992). Data reduction and error analysis for the physical sciences (2nd ed.), WCB/McGraw-Hill, Boston.

Da Silva, W. P., Silva, C. M. D. P. S., Silva, D. D. P. S., Neves, G. A., \& Lima, A. G. B. (2010). Mass and heat transfer study in solids of revolution via numerical simulations using finite volume method and generalized coordinates for the Cauchy boundary condition. International Journal of Heat and Mass Transfer, 53(5-6), 1183-1194. https://doi.org/10.1016/j.jheatmasstransfer.2009.10.028

Da Silva, W. P., Silva, C. M. D. P. S., \& Lins, M. A. A. (2011). Determination of expressions for the thermal diffusivity of canned foodstuffs by the inverse method and numerical simulations of heat penetration. International Journal of Food Science and Technology, 46(4), 811-818. https://doi.org/10.1111/j.1365-2621. 2011.02552.x

Da Silva, W. P., Silva, C. M. D. P. S., Precker, J. W., Gomes, J. P., Nascimento, P. L., \& Silva, L. D. (2012). Diffusion models for the description of seedless grape drying using analytical and numerical solutions. Agricultural Sciences, 3(4), 545-556. https://doi.org/10.4236/as.2012.34065

Da Silva, W. P., Silva, C. M. D. P. S., Souto, L. M., Moreira, I. S., \& Silva, E. C. O. (2018). Mathematical model for determining thermal properties of whole bananas with peel during the cooling process. Journal of Food Engineering, 227, 11-17. https://doi.org/10.1016/j.jfoodeng.2018.02.003

Dincer, I. (1995). Thermal cooling data for figs exposed to air cooling. International Communications in Heat Mass Transfer, 22(4), 559-566. https://doi.org/10.1016/0735-1933(95)00041-V 
Erdogdu, F., Linke, M., Praeger, U., Geyer, M., \& Schlüter, O. (2014). Experimental determination of thermal conductivity and thermal diffusivity of whole green (unripe) and yellow (ripe) Cavendish bananas under cooling conditions. J. Food Eng., 128, 46-52. https://doi.org/10.1016/j.jfoodeng.2013.12.010

Fricke, B. A., \& Becker, B. R. (2001). Evaluation of thermophysical property models for foods. HVAC R Res., 7(4), 311-330. https://doi.org/10.1080/10789669.2001.10391278

Levenberg, K. (1944). A method for the solution of certain problems in least squares. Q. Appl. Math, 2(2), 164-168. https://doi.org/10.1090/qam/10666

Marquardt, D. W. (1963). An algorithm for least-squares estimation of nonlinear parameters. J. Soc. Ind. Appl. Math., 11(2), 431-441. https://doi.org/10.1137/0111030

Patankar, S. V. (1980). Numerical heat transfer and fluid flow. New York: Hemisphere Publishing Corporation.

Press, W. H., Teukolsky, S. A., Vetterling, W. T., \& Flannery, B. P. (1996). Numerical Recipes in Fortran 77. The Art of Scientific Computing. New York: Cambridge University Press.

Silva, W. P., Silva, C. M. D. P. S., Farias, V. S. O., \& Silva, D. D. P. S. (2010). Calculation of the convective heat transfer coefficient and cooling kinetics of an individual fig fruit. Heat Mass Transfer, 46(3), 371-380. https://doi.org/10.1007/s00231-010-0577-7

Silva, W. P., Carmo, J. E. F., Silva, C. M. D. P. S., \& Aragão, R. F. (2011). Determination of Convective Heat Transfer Coefficient during Cooling of an Individual Strawberry Fruit Using Different Methods. International Review of Chemical Engineering, 3(2), 233-240.

Silva, W. P., Silva, C. M. D. P. S., \& Gama, F. J. A. (2012). An improved technique for determining transport parameters in cooling processes. Journal of Food Engineering, 111, 394-402. https://doi.org/10.1016/ j.jfoodeng.2012.02.003

Sweat, V. E. (1986). Thermal properties of foods. In M. A. Rao, \& S. S. H. Rizvi (Eds.), Engineering properties of foods (pp. 49-87). Marcel Dekker, New York.

Taylor, J. R. (1997). An introduction to error analysis (2nd ed.). University Science Books, Sausalito.

\section{Copyrights}

Copyright for this article is retained by the author(s), with first publication rights granted to the journal.

This is an open-access article distributed under the terms and conditions of the Creative Commons Attribution license (http://creativecommons.org/licenses/by/4.0/). 